\title{
Einstein Tensor and Generalizations of Birkhoff's Theorem^
}

\author{
Hubert GOENNER \\ Institut für Theoretische Physik, Universität Göttingen
}

Received June 20, 1969

\begin{abstract}
The Einstein tensors of metrics having a 3-parameter group of (global) isometries with 2-dimensional non-null orbits $G_{3}(2, s / t)$ are studied in order to obtain algebraic conditions guaranteeing an additional normal Killing vector. It is shown that Einstein spaces with $G_{3}(2, s / t)$ allow a $G_{4}$. A critical review of some of the literature on Birkhof's theorem and its generalizations is given.
\end{abstract}

\section{Introduction}

Birkhoff's theorem is stated, usually, in one of the following forms [1, 2]: (1) The most general spherically symmetric solution of Einstein's vacuum field equations is the Schwarzschild exterior solution [3] and (2) any spherically symmetric solution of Einstein's vacuum field equations is static [4].

A metric is defined to be spherically symmetric if it allows as a group of isometries the rotation group $0(3, R)$ acting on spacelike 2-dimensional orbits. All corresponding Killing vectors are normal (hypersurface orthogonal). The metric is defined to be static if it allows a timelike and normal Killing vector

$$
\xi_{(\alpha ; \beta)}=0, \quad \xi_{[\alpha} \xi_{\beta, \gamma]}=0, \quad \xi^{\alpha} \xi^{\beta} g_{\alpha \beta}>0 .
$$

Conditions (1) are local and, in general, limited to certain coordinate neighborhoods of the Riemannian manifold. For example, the Schwarzschild exterior solution (in Schwarzschild coordinates)

$$
d s^{2}=\left(1-\frac{2 m}{r}\right) d t^{2}-\left(1-\frac{2 m}{r}\right)^{-1} d r^{2}-r^{2}\left(d \theta^{2}+\sin ^{2} \theta d \varphi^{2}\right)
$$

allows a timelike normal Killing vector $\xi^{\alpha}=\delta_{0}^{\alpha}$ only for the range $r>2 m$. Beyond the null surface $r=2 m$, this Killing vector stays normal yet becomes spacelike. As Eq. (1) shows, this behavior is independent of the coordinates chosen.

* This work was started at the Department of Physics, Temple University, Philadelphia, Pa., and supported there by the Aerospace Research Laboratories of the Office of Aerospace Research, U.S.A.F. 
We suggest the following formulation of Birkhoff's theorem:

1. There is a unique 1-parameter family of spherically symmetric solutions of Einstein's vacuum field equations, the Schwarzschild solutions.

2. For a certain range of the coordinates, the Schwarzschild metrics are static.

Corollary. Any spherically symmetric solution of Einstein's vacuum field equations allows a fourth normal Killing vector.

Thus, Birkhoff's theorem contains two aspects; a statement about the uniqueness of a certain class of vacuum solutions and a group theoretical result; if a vacuum solution allows the rotation group $0(3, R)$ as group of isometries $G_{3}$, it also allows a $G_{4}$.

Since Birkhoff's theorem was first advanced its realm of application has been extended. Global groups of isometries other than the rotation group have been included. The vacuum field equations have been replaced by the field equations with a cosmological constant and/or certain types of matter. These extensions are briefly reviewed in Section 3 .

In Section 2 we investigate systematically what classes of metrics allowing 3-parameter groups of isometries with 2-dimensional, nonnull orbits admit an additional Killing vector. The n.a.s. conditions for an additional Killing vector being provided by Killing's equations, we are mainly interested in equivalent algebraic conditions on the Einstein tensor (matter tensor). It would be convenient if -one were able to tell from the algebraic structure of the Einstein tensor and its symmetry properties whether there exists an additional Killing vector.

The investigations of Section 2 show that, in general, by taking into account the Plebaǹski types of the Einstein tensor only [5] it is impossible to establish a n.a.s. criterion for the existence of an additional Killing vector. This is indicated already by the work of Takeno on spherically symmetric metrics [6]. It may be possible to obtain such a criterion by using further second order invariants for the description of. the metric. It is doubtful, though, whether by this method one would gain an advantage over solving Killing's equations.

\section{Groups of Isometries, Einstein Tensor and Generalizations of Birkhoff's Theorem}

\section{a) 3-Parameter Groups of Isometries with 2-Dimensional Orbits}

It is known that Einstein spaces of Petrov type $D$ with an $r$-parameter group of isometries $G_{r}(r>4)$ admit also a $G_{r+1}$ (indeed, they admit the maximal group $G_{10}$ ) [7]. In looking for an extension of the group theoretical side of Birkhoff's theorem one might ask whether an Einstein 
space of type $D$ with a $G_{3}$ as (global) group of isometries admits also a $G_{4}$. In the following, we limit the question to the extent that only groups $G_{3}$ with 2-dimensional orbits are included. For groups with spacelike, timelike and null orbits the notations $G_{3}(2, s), G_{3}(2, t)$, and $G_{3}(2, n)$ are introduced. In a sense, the question above has been answered positively: An Einstein space with $G_{3}(2, s / t)$ admits an additional Killing vector [8]. (Metrics with $G_{3}(2, n)$ are ruled out; they have Petrov type $N$.) However, the proof given in [8] is incomplete. In the following, the additional arguments needed are supplied.

The groups $G_{3}(2, s / t)$ and the canonical forms of the metrics admitting them as groups of isometries were discussed recently [9]. Altogether, there are six $G_{3}(2, s / t)$ with four distinct Lie algebras $A_{3}$ (see Table 1, Appendix 1). For the infinitesimal generators of the algebra and the corresponding Killing vectors we use the notation $X_{a}=\xi_{a}^{\alpha} \frac{\partial}{\partial x^{\alpha}}$ $(a=1,2,3)$. All possible extensions by another generator $Y=\eta^{\alpha} \frac{\partial}{\partial x^{\alpha}}$ of the Lie algebras $A_{3}$ are considered. The various cases occuring are summarized in Table 2, Appendix 1. There are two types of extensions: (1) central extensions with

$$
\left[X_{a}, Y\right]=0 \quad(a=1,2,3),
$$

and (2) non-central extensions with

$$
\left[X_{a}, Y\right]=c_{a}^{b} X_{b}+d_{a} Y \quad(a=1,2,3),
$$

$G_{3}$ is maximal on a 2-dimensional space. Thus, the orbit of $G_{4}$ with Killing vectors $\xi_{a}^{\alpha}, \eta^{\alpha}$ is the topological product of the 2-dimensional orbit (whose tangent plane is spanned by $\xi_{a}^{\alpha}$ ) and the trajectory of $\eta^{\alpha}$.

For central extensions, the additional Killing vector $\eta^{\alpha}$ lies in a 2-dimensional plane orthogonal to the tangent space of the orbit of $G_{3}$. This follows from Eq. (3a) through $\left[X_{a}, Y\right]=\left(\begin{array}{ll}E \\ \xi_{a}\end{array} \eta^{\sigma}\right) \frac{\partial}{\partial x^{\sigma}}(E \equiv$ Lie derivative) and the fact that $G_{3}$ is maximal on the orbit. Consequently, $\eta^{\alpha}$ is normal. In the case of the algebras not centrally extended this does not hold in general. For the algebra with Kruchkovich-Petrov type I (PKI) [10] the structure relations determine $Y$ to be $x^{2} \frac{\partial}{\partial x^{3}}$. Again, with $G_{3}$ being maximal on the orbit, this case does not occur $\left(\eta^{\alpha}=0\right)$. For the algebra with PKV we obtain through the structure relations

$$
y=x^{2} \frac{\partial}{\partial x^{2}}+x^{3} \frac{\partial}{\partial x^{3}}+\eta^{0}\left(x^{0}, x^{1}\right) \frac{\partial}{\partial x^{0}}+\eta^{1}\left(x^{0}, x^{1}\right) \frac{\partial}{\partial x^{1}} .
$$


From Killing's equations one may convince oneself that, after a suitable coordinate transformation, $\eta^{0}=-\eta^{1}=1$. The Killing vector corresponding to $Y$

$$
\eta^{\alpha}=x^{2} \delta_{2}^{\alpha}+x^{3} \delta_{3}^{\alpha}+\delta_{0}^{\alpha}-\delta_{1}^{\alpha}
$$

is normal. Summarizing we may say: If a metric with a group of isometries $G_{3}(2, s / t)$ admits an additional Killing vector, it is normal.

\section{b) Canonical Forms of Metrics with $G_{3}(2, s / t)$}

The canonical forms of metrics with $G_{3}(2, s / t)$ as global groups of isometries are taken from [9]. For spacelike orbits $x^{0}=$ const, $x^{1}=$ const

$$
d s^{2}=e^{2 \alpha\left(x^{0}, x^{1}\right)}\left(d x^{0}\right)^{2}-e^{2 \beta\left(x^{0}, x^{1}\right)}\left(d x^{1}\right)^{2}-e^{2 \gamma\left(x^{0}, x^{1}\right)} d \omega^{2}(2,3) .
$$

For timelike orbits $x^{1}=$ const, $x^{3}=$ const

$$
d s^{2}=e^{2 \gamma\left(x^{1}, x^{3}\right)} d \omega^{2}(0,2)-e^{2 \beta\left(x^{1}, x^{3}\right)}\left(d x^{1}\right)^{2}-e^{2 \alpha\left(x^{1}, x^{3}\right)}\left(d x^{3}\right)^{2}
$$

with

and

$$
d \omega^{2}(a, b)=\left(d x^{a}\right)^{2} \pm \Sigma^{2}\left(x^{a}\right)\left(d x^{b}\right)^{2}
$$

$$
\Sigma\left(x^{a}\right)=\left\{\begin{array}{l}
\sin x^{a} \\
\sinh x^{a} \\
1
\end{array}\right.
$$

for (constant) positive, negative and zero curvature of the orbits, respectively, with the sign in (7a) chosen such that the signature of the metric is -2 .

We omit the class of metrics with canonical form (6) and coordinates $x^{0}$ and $x^{2}$ exchanged. The Killing vector $\xi^{\alpha}=\delta_{0}^{\alpha}$ of its $G_{3}(2, t)$ is timelike and normal.

Every 2-dimensional space being conformally flat, only two of the arbitrary functions $\alpha, \beta, \gamma$ in Eqs. (5), (6) are essential. Often, coordinates are introduced such that $\gamma=\log x^{1}$ (curvature coordinates [4], canonical coordinates [6]). However, as discussed in detail by Takeno for spherically symmetric metrics, certain classes of metrics do not allow such coordinates. Takeno's discussion being rather abstract, we give an example: a metric with $G_{3}(2, s)$ and an additional null Killing vector does not admit curvature coordinates. In the case of axially symmetric metrics, a similar situation was discussed by R. Hoffman [24].

We assume $\alpha$ and $\beta$ to be of class $C^{2}$, piecewise $C^{4}$. Thus, we may extend Takeno's assertion (again for spherically symmetric metrics) that it is always possible to introduce coordinates such that the canonical form of metrics with $G_{3}(2, s)$ is

$$
d s^{2}=2 e^{2 \alpha(u, v)} d u d v-e^{2 \gamma(u, v)} d \omega^{2}(2,3) .
$$

In further discussions we shall use Eq. (5') instead of Eq. (5). 
While metrics with $G_{3}(2, t)$ allow additional spacelike Killing vectors only, such a vector may have arbitrary space-time character for metrics with $G_{3}(2, s)$. From subsection a it follows that such an additional Killing vector is described by

and

$$
\eta^{\alpha}=a(u, v) \delta_{u}^{\alpha}+b(u, v) \delta_{v}^{\alpha}
$$

$$
\eta^{\alpha}=a\left(x^{1}, x^{3}\right) \delta_{1}^{\alpha}+b\left(x^{1}, x^{3}\right) \delta_{3}^{\alpha}
$$

for Eqs. $\left(5^{\prime}\right)$ and (6). From Killing's equations it follows that metrics with $G_{3}(2, s)$ and an additional non-null Killing vector $(a \cdot b \neq 0)$ have the canonical form

$$
d s^{2}=2 e^{2 \alpha(u \mp v)} d u d v-e^{2 \gamma(u \mp v)} d \omega^{2}(2,3)
$$

while metrics with $G_{3}(2, s)$ and an additional null Killing vector $(a \cdot b=0)$ $\eta^{\alpha}=\delta_{u}^{\alpha}$ may be written as

$$
d s^{2}=d u d v-e^{2 \gamma(v)} d \omega^{2}(2,3) .
$$

Another form is obtained from Eq. (11) by interchanging $u$ and $v$.

For metrics with $G_{3}(2, t)$ one obtains $\eta^{\alpha}=\delta_{1}^{\alpha}$ and

$$
d s^{2}=\left(x^{3}\right)^{2} d \omega^{2}(0,2)-e^{2 \beta\left(x^{3}\right)}\left(d x^{1}\right)^{2}-e^{2 \alpha\left(x^{3}\right)}\left(d x^{3}\right)^{2}
$$

as well as the line element obtained by interchange of coordinates $x^{1}$ and $x^{3}$. Finally, the metric with $G_{4}$ belonging to $A_{4}$ of PKV and whose additional Killing vector is given by Eq. (4) may be written as

$$
d s^{2}=e^{\alpha\left(x^{0}+x^{1}\right)}\left(d x^{0}\right)^{2}-e^{\beta\left(x^{0}+x^{1}\right)}\left(d x^{1}\right)^{2}-e^{2 x^{1}}\left[\left(d x^{2}\right)^{2}+\left(d x^{3}\right)^{2}\right] .
$$

\section{c) Einstein Tensor and Additional Killing Vector}

The Einstein tensors $G_{\alpha}{ }^{\beta}$ corresponding to the canonical forms Eqs. $\left(5^{\prime}\right)$ and (6) are given in Appendix 2. They each possess one double eigenvalue,

and

$$
\begin{aligned}
& \lambda_{1,2}=G_{2}{ }^{2}=G_{3}{ }^{3} \\
& \lambda_{1,2}=G_{0}{ }^{0}=G_{2}{ }^{2}
\end{aligned}
$$

respectively. Thus, the Plebaǹski types occuring are $[Z-\bar{Z}-2 S]_{[1-1-1]}$, $\left[T-2 S_{1}-S_{2}\right]_{[1-1-1]}$ and $[2 N-2 S]_{[2-1]}$ for metrics with $G_{3}(2, s)$ and $\left[2 T-S_{1}-S_{2}\right]_{[1-1-1]}$ for $G_{3}(2, t)$. In the case of metrics with $G_{3}(2, s)$, the remaining eigenvalues of $G_{\alpha}^{\beta}$ are given by

$$
\lambda_{3,4}=G_{(u)}^{(u)} \pm\left(G_{(u)}{ }^{(v)} G_{(v)}{ }^{(u)}\right)^{\frac{1}{2}} .
$$

For an additional Killing vector (8) we may rewrite the off-diagonal components of $G_{\alpha}^{\beta}$ by using Killing's equations:

$$
G_{(u)}^{(v)}=\psi b^{2} e^{-2 \alpha}, \quad G_{(v)}^{(u)}=\psi a^{2} e^{-2 \alpha} .
$$


Here, $\psi=\psi(u, v)$ is an arbitrary real function. Thus, from Eq. (16)

$$
\lambda_{3,4}=G_{(u)}^{(u)} \pm 2 \psi a \cdot b e^{-2 \alpha} .
$$

Eqs. (17) and (18) hold for both $a \cdot b \neq 0$ (non-null Killing vector) and $a \cdot b=0$ (null Killing vector). In the latter case, for $a \neq 0, b=0$

$$
\psi=-\gamma_{, v v}-\left(\gamma_{, v}\right)^{2} .
$$

(A similar relation holds for $a=0, b \neq 0$.) From Eq. (18) it is seen that metrics with $G_{3}(2, s)$ and additional Killing vector cannot be of type $[Z-\bar{Z}-2 S]_{[1-1-1]}$. Eqs. (10), (11), and (A2.1) show that $\left[T-2 S_{1}-S_{2}\right]_{[1-1-1]}$ and $[2 N-2 S]_{[2-1]}$ (and more degenerate types) may occur.

From Eqs. (17), (18), (19) and the remaining components of $G_{\alpha}{ }^{\beta}$ (see Appendix 2) as well as from corresponding considerations for the metrics with $G_{3}(2, t)$ one may conclude:

(1) If a metric with $G_{3}(2, s / t)$ allows an additional non-null Killing vector, this is an eigenvector of $G_{\alpha}{ }^{\beta}$. An additional null Killing vector is eigenvector of $G_{\alpha}{ }^{\beta}$ only if $\psi$ of Eq. (19) is zero.

By checking the components of the Riemann curvature tensor one obtains:

(2) A non-flat vacuum solution with $G_{3}(2, s / t)$ as group of isometries cannot have an additional null Killing vector.

Comparing Eqs. (10), (11), and (12) with the canonical forms of metrics whose Einstein tensor has two double eigenvalues or one quadruple eigenvalue (see Appendix 2) we may state:

(3) Metrics with $G_{3}(2, s)$ whose corresponding Einstein tensors are of Plebaǹski type $[2 T-2 S]_{[1-1]}$ and $[4 T]_{[1]}$ allow a $G_{4}$.

(4) Metrics with $G_{3}(2, s)$ whose corresponding Einstein tensors are of Plebaǹski type $[2 N-2 S]_{[2-1]}$ and $[4 N]_{[2]}$ may or may not allow an additional Killing vector.

If they do admit an additional Killing vector it is a null vector. The canonical form of such metrics is given by Eq. (11). Type $[4 N]_{[2]}$ occurs if $\gamma_{, v v}+\left(\gamma_{, n}\right)^{2} \neq 0$ and $\Sigma\left(x^{0}\right)=1$. Such metrics are non-flat with $R=0$ and $C=0$. Statement (3) is contained in [9] already while (4) should be added to the relevant discussions of [9] and [19]. It was not obtained there, because these investigations were based on canonical coordinates.

(5) Metrics with $G_{3}(2, t)$ whose corresponding Einstein tensors are of type $[4 T]_{[1]}$ allow a $G_{4}$ while those of type $[2 T-2 S]_{[1-1]}$ need not admit a further Killing vector.

An example of such a metric with $G_{3}(2, t)$ of type $[2 T-2 S]_{[1-1]}$ is provided by Eq. (5.21) (with $k=0$ ) of [9]. 
Defining an Einstein space to be a space whose Einstein tensor is proportional to the first fundamental form, there are then two types of Einstein spaces with $G_{3}(2, s)$ :

$$
d s^{2}=2 e^{\alpha(u, v)} d u d v-c^{2} d \omega^{2}(2,3)
$$

with $c$ constant and

$$
2 \alpha_{, u v} e^{-2 \alpha}+\Sigma^{-1} \Sigma_{, 22} \cdot \frac{1}{c^{2}}=0 .
$$

Furthermore,

$$
d s^{2}=F_{, z} d u d v-F^{2} d \omega^{2}(2,3)
$$

where $F=F(z), z=u \pm v$ satisfies

$$
\pm\left(F_{, z}\right)^{-1}\left(\log F_{, z}\right)_{, z z} \mp 2 F^{-2} F_{, z}+\Sigma^{-1} \Sigma_{, 22} F^{-2}=0 .
$$

By comparison with Eq. (10) one knows that the metric (21a) allows a $G_{4}$. From the general solution of the hyperbolic differential equation (20b) given in [22] one may draw the same conclusion for the metric (20a). However, in order to see this one need not solve Eq. (20b). Following an idea of Sommerfeld's [23] we calculate the Gaussian curvature $K$ of the 2-space $d \sigma^{2}=2 e^{2 \alpha(u, v)} d u d v$. It is given by Eq. (20b) with $K=\Sigma^{-1} \Sigma_{, 22} \cdot \frac{1}{c^{2}}=$ constant. A 2-space of constant curvature allows the maximal group of isometries, i.e. a $G_{3}$. Thus, an Einstein space with $G_{3}(2, s)$ and line element (20a) allows a $G_{6}$.

For Einstein spaces with $G_{3}(2, t)$ the same argument is valid although Eq. (20b) is to be replaced by an elliptical partial differential equation (see Eq. (A 2.11) of Appendix). Summarizing we may say:

(6) A non-flat Einstein space with $G_{3}(2, s / t)$ as group of isometries allows a $G_{4}$.

We have not investigated whether all metrics with $G_{3}(2, s)$ and remaining degeneracies $[3 T-S]_{[1-1]}$ and $[T-3 S]_{[1-1]}\left(G_{3}(2, t)\right.$ with $[3 T-S]_{[1-1]}$ ) do allow a $G_{4}$. However, statements (4) and (5) make clear already that the Plebaǹski type alone does not determine whether an additional Killing vector is admitted or not. In order to find a n.a.s. criterion one ought to investigate more of the second order differential invariants for the canonical forms (5') and (6) [6].

\section{Comments on the Literature on Birkhoff's Theorem}

\section{a) Reexamination of the Original Proofs}

Birkhoff's theorem has been discovered, independently, by Jebsen, Alexandrow, and Birkhoff $[1,2]$. The proof given by Birkhoff seems to be the most general. He proceeded from the canonical form (5) with 
$\alpha=\beta$ (i.e. essentially from Eq. $\left.\left(5^{\prime}\right)\right)$ while Jebsen and Alexandrow chose canonical coordinates. Thus they excluded the possibility of an additional null Killing vector. This does not affect the validity of the corollary to Birkhoff's theorem given in Section 1; as shown in Section 2c, there are no spherically symmetric Einstein spaces with an additional null Killing vector.

Eiesland presented another proof of Birkhoff's theorem by investigating Killing's equations for the canonical form Eq. (5) and a Killing vector (8) [11]. By using the redundancy inherent in the functions $\alpha, \beta, \gamma$ of (5) he was able to rewrite Killing's equations, for $\gamma \neq$ const, as follows:

$$
\begin{aligned}
G_{(0)}{ }^{(0)}-G_{(1)}^{(1)}+\frac{d \psi}{d \gamma}\left[\left(\frac{d \gamma}{d x^{0}}\right)^{2} e^{-2 \alpha}+\left(\frac{d \gamma}{d x^{1}}\right)^{2} e^{-2 \beta}\right] & =0 \\
G_{(0)}{ }^{(1)}-\frac{d \psi}{d \gamma} \cdot \frac{\partial \gamma}{\partial x^{0}} \cdot \frac{\partial \gamma}{\partial x^{1}} \cdot e^{-2 \beta} & =0
\end{aligned}
$$

where $\psi=\psi(\gamma)$ is arbitrary. The invariant components of $G_{(\alpha)}^{(\beta)}$ used in Eq. (21) are connected to the components given in Eq. (A2.1) of Appendix 2 by

$$
\begin{aligned}
& G_{(0)}{ }^{(0)}=\frac{1}{2}\left(2 G_{(u)}{ }^{(u)}+G_{(u)}{ }^{(v)}+G_{(v)}{ }^{(u)}\right), \\
& G_{(1)}{ }^{(1)}=\frac{1}{2}\left(2 G_{(u)}{ }^{(u)}-G_{(u)}{ }^{(v)}-G_{(v)}{ }^{(u)}\right), \\
& G_{(0)}{ }^{(1)}=\frac{1}{2}\left(G_{(v)}{ }^{\left({ }^{\prime}\right)}-G_{(u)}{ }^{(v)}\right) .
\end{aligned}
$$

One may verify that Eq. (21) holds for all metrics with $G_{3}(2, s / t)$. In the latter case, all indices 0 in Eq. (21) should be replaced by an index 3 . Thus, statement (6) of Section $2 \mathrm{c}$ may be derived immediately from Eq. (21). The case $\gamma=$ const. was also treated by Eiesland and shown to be static. Later, it was discussed by Bonnor [12] and discarded as unphysical.

Thus far, it was assumed that the functions $\alpha, \beta, \gamma$ appearing in Eqs. (5), (5'), and (6) are of class $C^{2}$, piecewise $C^{4}$. This guarantees the introduction of curvature coordinates or the removal of an off-diagonal term in the line element. The conditions usually imposed on the metric tensor require $g_{\alpha \beta}$ to be only of class $C^{1}$, piecewise $C^{3}$ [13]. Using this apparent discrepancy, Petrov suggested that Birkhoff's theorem might not hold for metrics of low differentiability and constructed an example for a spherically symmetric solution of the vacuum field equations seemingly different from the Schwarzschild metric [14]. In the ensuing discussion, Hamoui removed the discrepancy between the various differentiability requirements [15], while Bergmann, Cahen, and Komar 
showed that Petrov's example represented Schwarzschild's metric in coordinates of low class [16]. They also proved that spherically symmetric shock waves cannot exist. This result had been obtained previously by Papapetrou [17].

\section{b) Extensions to Non-Vacuum Field Equations}

Eiesland seems to have been the first to extend Birkhoff's theorem beyond its application to vacuum solution [11]. From Eq. (21) he concluded that spherically symmetric Einstein spaces are static. As special examples, he mentioned de Sitter space and the Reissner-Nordström metric. In generalizing this result, Hoffmann showed that Birkhoff's theorem, in the formulation given in Section 1, holds for the combined Einstein-Maxwell equations (including a cosmological term) [18], with a unique 3-parameter solution. Recently, Plebaǹski and Stachel noted that spherically symmetric metrics with Einstein tensors of Plebanski type $[2 T-2 S]_{[1-1]}$ and $[4 T]_{[1]}$ are static $[19]$.

\section{c) Extensions Beyond Spherical Symmetry}

Taub showed that one may replace the rotation group $0(3, R)$ in Birkhoff's theorem by other groups of isometries, i.e. by $G_{3}(2, s)$ [20] (see Table 1 of Appendix 1). In all three cases, there is a unique 1-parameter family of solutions of the vacuum field equations.

A further generalization was obtained by Cahen and Debever by including metrics with $G_{3}(2, t)$ and considering Einstein spaces: An Einstein space with $G_{3}(2, s / t)$ allows also a $G_{4}$ [8]. However, Cahen and Debever define an Einstein space by $R_{\alpha \beta}=0$ rather than by $S_{\alpha \beta}=R_{\alpha \beta}$ $-\frac{1}{4} R g_{\alpha \beta}=0$ (Eq. (3.1 c) of [8] is equivalent to $S_{\alpha \beta}=0$ while Eq. (3.1 d) gives $R=0$.) Thus, in fact, the authors have extended the results of Birkhoff and Taub to include metrics with $G_{3}(2, t)$. The extension to Einstein spaces with $R \neq 0$ for $G_{3}(2, s / t)$ is given in Section $2 \mathrm{c}$.

Acknowledgements. The author wishes to thank Dr. P. Havas, Philadelphia, for suggesting this investigation and for his kind hospitality at Temple University. He is grateful to Drs. J. Stachel, Boston University, for critical comments, J. LeRoy, Free University of Brussels, for pointing out Ref. [22], and R. B. Hoffman, Franklin and Marshall College, Lancaster, for clearing up the relationship with Ref. [24].

\section{Appendix}

a) Algebras $A_{3}$ Belonging to the Groups $G_{3}(2, s / t)$ and Algebras $A_{4} \supset A_{3}$

As discussed in [9], within the Bianchi classification of 3-dimensional algebras $A_{3}[21]$ there are altogether four different such Lie algebras 
Table 1. Lie algebras $A_{3}$ corresponding to groups $G_{3}(2, s / t)$. In column 1 , the numbers of the Bianchi classification are given as used in [7]. The three different orbits of type VIII belong to three non-equivalent groups having the same algebra

\begin{tabular}{|c|c|c|c|}
\hline & Lie algebra $A_{3}$ & Group orbit & \\
\hline$I X$ & $\begin{array}{l}{\left[X_{1}, X_{2}\right]=X_{3},\left[X_{2}, X_{3}\right]=X_{1}} \\
{\left[X_{3}, X_{1}\right]=X_{2}}\end{array}$ & $\begin{array}{l}\text { spacelike, positive } \\
\text { (constant) curvature }\end{array}$ & \multirow{2}{*}{$G_{3}(2, s)$} \\
\hline VIII & $\begin{array}{l}{\left[X_{1}, X_{2}\right]=X_{1},\left[X_{2}, X_{3}\right]=X_{3}} \\
{\left[X_{3}, X_{1}\right]=-2 X_{2}}\end{array}$ & $\begin{array}{l}\text { spacelike, negative } \\
\text { (constant) curvature } \\
\text { timelike, positive } \\
\text { and negative } \\
\text { (constant) curvature }\end{array}$ & \\
\hline VII $(q=0)$ & $\begin{array}{l}{\left[X_{1}, X_{2}\right]=0, \quad\left[X_{2}, X_{3}\right]=-X_{1}} \\
{\left[X_{3}, X_{1}\right]=-X_{2}}\end{array}$ & $\begin{array}{l}\text { spacelike, zero } \\
\text { curvature }\end{array}$ & $G_{3}(2, s)$ \\
\hline $\mathrm{VI}(q=-1)$ & $\begin{array}{l}{\left[X_{1}, X_{2}\right]=0, \quad\left[X_{2}, X_{3}\right]=-X_{2}} \\
{\left[X_{1}, X_{3}\right]=X_{1}}\end{array}$ & $\begin{array}{l}\text { timelike, zero } \\
\text { curvature }\end{array}$ & $G_{3}(2, t)$ \\
\hline
\end{tabular}

Table 2. Extensions of Lie algebra $A_{3}$ of Table 1. In column 1, the numbers of the PetrovKruchkovich classification, in column 2, the numbers of the Bianchi classification are given [7]. The assumption that $\eta^{\alpha}$ belonging to $Y$ be a Killing vector imposes further conditions on the still undetermined functions $a^{0}$ and $a^{1}$

\begin{tabular}{|c|c|c|c|}
\hline & & $\begin{array}{l}\text { Additional commutation } \\
\text { relations }\end{array}$ & Representation of $Y$ \\
\hline \multirow{4}{*}{$=0$} & IX & {$\left[X_{a}, Y\right]=0$} & $Y=a^{0}\left(x^{0}, x^{1}\right) \frac{\partial}{\partial x^{0}}+a^{1}\left(x^{0}, x^{1}\right) \frac{\partial}{\partial x^{1}}$ \\
\hline & VIII & {$\left[X_{a}, Y\right]=0$} & same \\
\hline & $\begin{array}{l}\text { VII } \\
q=0\end{array}$ & {$\left[X_{a}, Y\right]=0$} & same \\
\hline & $\begin{array}{l}\text { VII } \\
q=0\end{array}$ & $\begin{array}{l}{\left[X_{1}, Y\right]=X_{1},\left[X_{2}, Y\right]=X_{2}} \\
{\left[X_{3}, Y\right]=0}\end{array}$ & $\begin{aligned} Y= & x^{2} \frac{\partial}{\partial x^{2}}+x^{3} \frac{\partial}{\partial x^{3}}+a^{0}\left(x^{0}, x^{1}\right)- \\
& +a^{1}\left(x^{0}, x^{1}\right) \frac{\partial}{\partial x^{1}}\end{aligned}$ \\
\hline \multirow[t]{3}{*}{$\xi-1, e=0$} & $\begin{array}{l}\mathrm{VI} \\
q=-1\end{array}$ & {$\left[X_{a}, Y\right]=0$} & $Y=a^{0}\left(x^{0}, x^{1}\right) \frac{\partial}{\partial x^{0}}+a^{1}\left(x^{0}, x^{1}\right) \frac{\partial}{\partial x^{1}}$ \\
\hline & $\begin{array}{l}\text { VI } \\
q=-1\end{array}$ & $\begin{array}{l}{\left[X_{1}, Y\right]=X_{2},\left[X_{2}, Y\right]=0} \\
{\left[X_{3}, Y\right]=2 Y}\end{array}$ & $Y=x^{2} \frac{\partial}{\partial x^{2}}$ \\
\hline & II & {$\left[X_{a}, Y\right]=0$} & $Y=a^{0}\left(x^{0}, x^{1}\right) \frac{\partial}{\partial x^{0}}+a^{1}\left(x^{0}, x^{1}\right) \frac{\partial}{\partial x^{1}}$ \\
\hline
\end{tabular}


corresponding to groups with 2-dimensional spacelike or timelike orbits. For the convenience of the reader we reproduce this list.

The following table summarizes all 4-dimensional Lie-algebras $A_{4}$ containing the $A_{3}$ 's of Table 1 as subalgebras. This table has been compiled by searching through the classification of 4-dimensional Lie algebras (over the real numbers) by Kruchkovich and Petrov [10] for such subalgebras.

\section{b) Einstein Tensor of Canonical Forms (5'), (6)}

The components of the Einstein tensor are refered to an orthonormal tetrad $e_{(\sigma)}^{\alpha}$.

$$
\begin{gathered}
\text { A. Canonical Form } \left.\left(5^{\prime}\right) \text { (Metrics with } G_{3}(2, s)\right) \\
e_{(u)}^{\sigma}=1 / \sqrt{2}\left(\delta_{u}^{\sigma}+\delta_{v}^{\sigma}\right) e^{-\alpha}, \quad e_{(v)}^{\sigma}=1 / \sqrt{2}\left(\delta_{u}^{\sigma}-\delta_{v}^{\sigma}\right) e^{-\alpha}, \quad e_{(2)}^{\sigma}=e^{-\gamma} \delta_{2}^{\sigma}, \\
e_{(3)}^{\sigma}=e^{-\gamma} \Sigma^{-1} \delta_{3}^{\sigma} \\
G_{(u)}^{(u)}=G_{(v)}^{(v)}=\left(4 \gamma_{, u} \gamma_{, v}+2 \gamma_{, u v}\right) e^{-2 \alpha}-\Sigma^{-1} \Sigma_{, 22} e^{-2 \gamma} \\
G_{(u)}^{(v)}=-2\left(\gamma_{, u u}+\left(\gamma_{, u}\right)^{2}-2 \alpha_{, u} \gamma_{, u}\right) e^{-2 \alpha} \\
G_{(v)}^{(u)}=-2\left(\gamma_{, v v}+\left(\gamma_{, v}\right)^{2}-2 \alpha_{, v} \gamma_{, v}\right) e^{-2 \alpha} \\
G_{(2)}^{(2)}=G_{(3)}^{(3)}=2\left(\alpha_{, u v}+\gamma_{, u v}+\gamma_{, u} \gamma_{, v}\right) e^{-2 \alpha}
\end{gathered}
$$

The curvature scalar $R=R_{\sigma}{ }^{\sigma}$ and conform invariant $C=\left[3 C_{\alpha \beta \gamma \delta} C^{\alpha \beta \gamma \delta}\right]^{\frac{1}{2}}$ are given by

$$
\begin{aligned}
& R=-4\left(\alpha_{, u v}+2 \gamma_{, u v}+3 \gamma_{, u} \gamma_{, v}\right) e^{-2 \alpha}+2 \Sigma^{-1} \Sigma_{, 22} e^{-2 \gamma}, \\
& C=2\left(\alpha_{, u v}-\gamma_{, u v}\right) e^{-2 \alpha}-\Sigma^{-1} \Sigma_{, 22} e^{-2 \gamma} .
\end{aligned}
$$

There are three classes of metrics whose Einstein tensor has two double eigenvalues:

$$
\begin{aligned}
d s^{2} & =2 e^{2 \alpha(u, v)} d u d v-c^{2} d \omega^{2}(2,3), \\
d s^{2} & =2 e^{2 \alpha(u, v)} d u d v-v^{2} d \omega^{2}(2,3), \\
d s^{2} & =2 F_{, u} d u d v-F^{2}(u, v) d \omega^{2}(2,3)
\end{aligned}
$$

and the metrics obtained by interchanging $u$ and $v$.

A quadruple eigenvalue exists if, in addition to $G_{(u)}{ }^{(v)} G_{(v)}{ }^{(u)} \neq 0$, the relation holds:

$$
2\left(\alpha_{, u v}-\gamma_{, u} \gamma_{, v}\right) e^{-2 \alpha}+\Sigma^{-1} \Sigma_{, 22} e^{-2 \gamma}=0 .
$$

Thus, the metric (A2.3) is an Einstein space if

$$
2 \alpha_{, u v} e^{-2 \alpha}+\Sigma^{-1} \Sigma_{, 22} \cdot \frac{1}{c^{2}}=0 .
$$

As was seen in subsection $2 \mathrm{c}$ the general solution of this equation is such that the corresponding metric allows additional Killing vectors. 
The only possible Einstein space of type Eq. (A 2.4) is flat space time. Finally, if the metric (A 2.5) is to be an Einstein space, one obtains from Eq. (A2.1) that $F_{, v}\left(F_{, u}\right)^{-1}=f(u)$. This is solved by $F=F(z)$ with $z=v+\int^{u} \frac{d y}{f(y)}$. By introducing the new coordinate $\bar{u}=\int^{u} \frac{d y}{f(y)}$ one arrives at Eq. (20b) of Section $2 c$.

B. Canonical Form (6) (Metrics with $G_{3}(2, t)$ )

The Einstein tensor for the metrics with $G_{3}(2, t)$ was given in [9]. For the convenience of the reader we reproduce the expressions for the special choice of canonical coordinates $\gamma=\log x^{3}$ :

$$
\begin{aligned}
& G_{(0)}{ }^{(0)}=G_{(2)}{ }^{(2)}=e^{-2 \alpha}\left(\frac{\dot{\alpha}-\dot{\beta}}{x^{3}}-\ddot{\beta}+\dot{\alpha} \dot{\beta}-\dot{\beta}^{2}\right)+e^{-2 \beta}\left(\alpha^{\prime} \beta^{\prime}-\alpha^{\prime 2}-\alpha^{\prime \prime}\right), \\
& G_{(1)}^{(1)}=e^{-2 \alpha}\left(2 \frac{\dot{\alpha}}{x^{3}}-\frac{1}{\left(x^{3}\right)^{2}}\right)+\frac{1}{\left(x^{3}\right)^{2}} \Sigma^{-1} \Sigma_{, 22}, \\
& G_{(3)}{ }^{(3)}=e^{-2 \alpha}\left(-2 \frac{\dot{\beta}}{x^{3}}-\frac{1}{\left(x^{3}\right)^{2}}\right)+\frac{1}{\left(x^{3}\right)^{2}} \Sigma^{-1} \Sigma_{, 22} . \\
& G_{(1)}{ }^{(3)}=-2 e^{-(\alpha+\beta)} \cdot \alpha^{\prime} / x^{3} .
\end{aligned}
$$

The tetrad used in Eq. (A 2.7) is

$$
e_{(0)}^{\sigma}=e^{-\gamma} \delta_{2}^{\sigma}, \quad e_{(1)}^{\sigma}=e^{-\beta} \delta_{1}^{\sigma}, \quad e_{(2)}^{\sigma}=e^{-\gamma} \Sigma^{-1} \delta_{0}^{\sigma}, \quad e_{(3)}^{\sigma}=e^{-\alpha} \delta_{3}^{\alpha} .
$$

Furthermore,

$$
\begin{aligned}
R= & 2 e^{-2 \alpha}\left(\frac{1}{\left(x^{3}\right)^{2}}-2 \frac{\dot{\alpha}-\dot{\beta}}{x^{3}}+\ddot{\beta}-\dot{\alpha} \dot{\beta}+\dot{\beta}^{2}\right)+2 e^{-2 \beta}\left(\alpha^{\prime \prime}+\alpha^{\prime 2}-\alpha^{\prime} \beta^{\prime}\right) \\
& -\frac{2}{\left(x^{3}\right)^{2}} \Sigma^{-1} \Sigma_{, 22} \\
C= & e^{-2 \alpha}\left(\frac{1}{\left(x^{3}\right)^{2}}+\frac{\dot{\alpha}-\dot{\beta}}{x^{3}}+\ddot{\beta}-\dot{\alpha} \dot{\beta}+\dot{\beta}^{2}\right)+e^{-2 \beta}\left(\alpha^{\prime \prime}+\alpha^{\prime 2}-\alpha^{\prime} \beta^{\prime}\right) \\
& -\frac{1}{\left(x^{3}\right)^{2}} \Sigma^{-1} \Sigma_{, 22}
\end{aligned}
$$

where the dot and dash indicate $\partial / \partial x^{3}$ and $\partial / \partial x^{1}$, respectively.

A second double eigenvalue exists if

$$
2 \alpha^{\prime}=(\alpha+\beta)^{\cdot} e^{\beta-\alpha} .
$$


For a quadruple eigenvalue one obtains the additional condition

$$
\begin{aligned}
e^{-2 \alpha}\left(\frac{1}{\left(x^{3}\right)^{2}}+\dot{\alpha} \dot{\beta}-\dot{\beta}^{2}-\ddot{\beta}\right) & +e^{-2 \beta}\left(\alpha^{\prime} \beta^{\prime}-\alpha^{\prime 2}-\alpha^{\prime \prime}\right) \\
& -\frac{1}{\left(x^{3}\right)^{2}} \Sigma^{-1} \Sigma_{, 22}=0 .
\end{aligned}
$$

If the metric with canonical form (6) is to be an Einstein space, we obtain from Eq. (A2.7) $\alpha^{\prime}=0,(\alpha+\beta)^{\circ}=0$. By comparison with Eq. (12), it is seen that the first equation guarantees an additional Killing vector. The second one, together with (A2.7), provides a unique 2-parameter family of solutions

with

$$
d s^{2}=\left(x^{3}\right)^{2} d \omega^{2}(0,2)-f\left(d x^{1}\right)^{2}-f^{-1}\left(d x^{3}\right)^{2}
$$

$$
f=\Sigma^{-1} \Sigma_{, 22}+\frac{a}{x^{3}}+b\left(x^{3}\right)^{2} .
$$

If canonical coordinates are not admissible, i.e. for $\gamma=$ const. in Eq. (6), we set $\alpha=\beta$. Then, Eq. (A2.10) is to be replaced by

$$
e^{-2 \alpha}\left(\ddot{\alpha}+\alpha^{\prime \prime}\right)-\Sigma^{-1} \Sigma_{, 22} \cdot \frac{1}{c^{2}}=0 .
$$

\section{References}

1. Birkhoff, G. D.: Relativity and modern physics, p. 255. Cambridge: Harvard University Press 1923.

2. Jebson, J. T.: Ark. Mat. Astron. Fys. 15, 1 (1921). - Alexandrow, W.: Ann. Physik 72, 141 (1923).

3. Anderson, J. L.: Principles of relativity physics, p. 336. New York: Academic Press 1967.

4. Synge, J. L.: Relativity, the general theory, p. 276. Amsterdam: North-Holland Publ. Co. 1966.

5. Plebaǹski, J.: Acta Phys. Polon. 26, 963 (1964).

6. Takeno, H.: The theory of spherically symmetric space times (Scientific Report No. 5 of the Research Institute for Theoretical Physics, Hiroshima University, 1966).

7. Petrow, A. S.: Einstein-Räume, S. 190. German translation: Berlin: Akademie Verlag 1964.

8. Cahen, M., Debever, R.: Compt. Rend. 260, 815 (1965).

9. Goenner, H., Stachel, J.: Einstein tensor and 3-parameter groups of isometries with 2-dimensional orbits. Preprint 1968. (to appear in J. Math. Phys.)

10. Kruchkovich, G. I.: Uspekh. Mat. Nauk 9, 10 (1954); Mat. Sbornik 41, 209 (1957); Petrow, A. S., [7], p. 60.

11. Eiesland, J.: Am. Math. Soc. Trans. 27, 213 (1925).

12. Bonnor, W. B.: In: Recent developments in general relativity, p. 167. New York: Pergamon Press 1962.

13. Lichnerowicz, A.: Théories relativistes de la gravitation et de l'électromagnetisme, p. 5. Paris: Masson et Cie. 1955. 
14. Petrov, A. Z.: JETP 17, 1026 (1963).

15. Hamoui, A.: Compt. Rend. 258, 6085 (1964).

16. Bergmann, P. G., Cahen, M., Komar, A. B.: J. Math. Phys. 6, 1 (1965).

17. Papapetrou, A.: Compt. Rend. 257, 2616 (1963).

18. Hoffmann, B.: Quart. J. Math. 3, 226 (1932) and 4, 179 (1933); and in Ref. 12, p. 279.

19. Plebaǹski, J., Stachel, J.: J. Math. Phys. 9, 269 (1968).

20. Taub, A. H.: Ann. Math. 53, 472 (1951).

21. Bianchi, L.: Lezioni sulla teoria dei gruppi finiti di trasformazioni. Pisa: Spoerri 1918.

22. Goursat, E.: Cours d'analyse mathematique, 4 Ed., Tome 3, p. 71. Paris: GauthierVillars 1927.

23. Sommerfeld, A.: In: Encyklopädie der Mathematischen Wissenschaften, II, 7c, 553 (1916).

24. Hoffman, R. B.: J. Math. Phys. 10, 953 (1969).

\author{
H. Goenner \\ Institut für theoretische Physik \\ der Universität \\ 34 Göttingen, Bunsenstr. 9
}

